\title{
Nanostructured Materials Application in Construction of Microhumidity Sensors
}

\author{
Maxim V. Chuprin, Olga M. Ivanova, Sergey A. Krutovertsev, \\ Anatoly V. Shevchenko, Alla E. Tarasova \\ JSC "Ecological sensors and systems", 124460, Moscow, Zelenograd, Russia \\ boss@pnc.ru
}

\begin{abstract}
:
Currently low moisture content measurement remains one of the most urgent tasks. Besides, the requirements in respect of trace amounts of moisture become stricter for many processes. Operation principle of the microhumidity sensor is based on a relationship between a capacity of doped silica film and an amount of humidity that is sorbed by it. Characteristics of thin nanostructured modified films that were produced by a hydrolytic polycondensation from tetraethoxysilane based solutions were studied. There are many technological factors that influence on adsorption value of films, such as: solution content, film formation conditions, temperature rate and technological environment influence. Influence of technological parameters on sensor characteristics was investigated. Special algorithm of signal processing was developed for temperature effect compensation on sensor's capacity. High sensitivity enables to use the sensors to measure humidity content for especially pure gases used in microelectronic technology, active gases at humidity content up to $-80{ }^{\circ} \mathrm{C}$ by dew point and organic solvents.
\end{abstract}

Key words: microhumidity sensor, nanostructured film, sol-gel

\section{Introduction}

Currently, moisture content measurement remains one of the most urgent tasks, especially in case of low concentrations in gas environments and organic solvents, widely used in various industries. Besides, the requirements in respect of trace amounts of moisture become stricter for many processes. Moisture often decisively influences the quality and quantity of output products and its measurement, especially its trace amounts, is very common and relevant task.

Sorption methods have the best set of characteristics for measuring small moisture concentrations among the known method for moisture measurement [1-2]. Irrespective of how signal is converted and recorded, a basic element of sorption micro moisture sensor is material layer that can reversibly sorb water vapors under measurement conditions in contact with test environment. Polymer or highly porous oxide films are usually used for these purposes [2-5]. The use of nanostructure films as sensitive layers of sorption sensors opens new prospects in sensor analysis. It is obvious that the higher the value of specific surface of the material, on the basis of which the film is formed, the higher efficiency of the sensor. Thus, the use of nano- and microporous materials for these purposes is an optimum engineering solution.

Nanoporous materials (pore diameter - less than $2 \mathrm{~nm}$ ), used in the structure of the sensor sensitive element, enables, on the one hand, to further increase the sensor sensitivity and, on the other hand, to reduce a hysteresis value in adsorption isotherm, and thereby to improve measurement accuracy.

This paper examines the nanoporous and nanostructure materials used as sensitive layers of micromoisture sensors. In particular, doped $\mathrm{SiO}_{x}$ films, produced by a hydrolytic polycondensation from tetraethyl orthosilicate (TEOS) based solutions, have been studied. Porous silica is a unique matrix material for chemical sensors, which has a lot of excellent physical and chemical properties. Sol-gel method enables to produce nanostructure sorbent films that have a developed specific surface with many active centers and high adsorption. Adsorption capacity of sorbent material determines the characteristics of sorption gas sensors and, in case of $\mathrm{SiO}_{x}$, depends on the number of hydroxyl groups that are present at its surface and are the centers of adsorption of water molecules. Incorporating 
the $\mathrm{KCl}, \mathrm{LiCl}$ and $\mathrm{P}_{2} \mathrm{O}_{5}$ hygroscopic additives into $\mathrm{SiO}_{\mathrm{x}}$ film increases the film sensitivity to water vapors. In this case, adsorption activity of $\mathrm{SiO}_{x}$ films depends on activating additive in a complex way.

\section{Experimental}

Analytical pure reagent TEOS was used to prepare the porous silica gel films. TEOS was thoroughly mixed with alcohol and water. Various quantities of hydrochloric acid were used to control the hydrolysis and polymerization of the TEOS to form a sol solution. Hygroscopic additives were added into base solution. All reagents were of analytical grade. We used solutions with different ageing time to deposition on wafer's surface.

Principle of operation of the micromoisture sensor is based on a relationship between a capacity of dielectric layer and an amount of moisture sorbed by such layer (see Fig.1). Glassceramics is used as substrate material. Electrode system based on nickel with vanadium sublayer with a thickness of $0.2-0.3$ $\mu \mathrm{m}$ is formed at its surface by the methods of thin-film technology. Sorbent film - doped nanostructure silicon oxide - was applied to the surface of the electrodes by hydrolytic polycondensation from TEOS solutions. The films were subsequently subjected to a multistep heat treatment at the temperature up to $413 \mathrm{~K}$ and higher. The drying process of gel was slow to prevent cracking of the films during drying. The developed technology of multistep temperature treatment allowed film properties stabilizing.

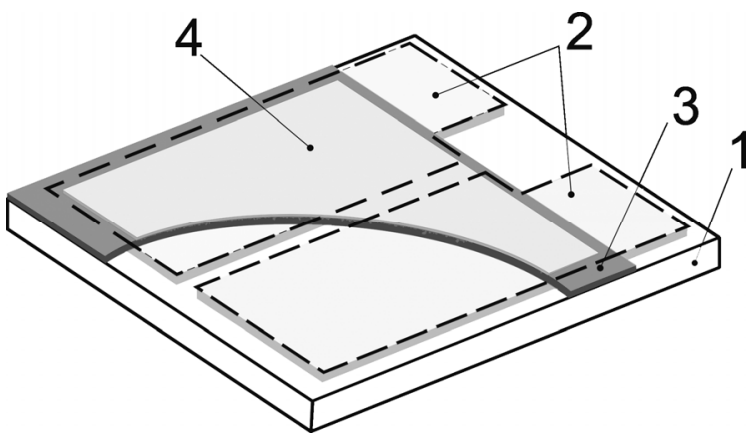

Fig. 1. A capacitive type humidity sensor: 1 substrate $\left(\mathrm{Al}_{2} \mathrm{O}_{3}\right) ; 2,3$ - electrodes (Ni); 4 nanostructured doped silica film; 5 -gold film.

Nano-size gold film is formed at the top of dielectric layer by thermal evaporation under vacuum. The film thickness $(\sim 10 \mathrm{~nm})$ is formed in such a way that the way is permeable for water molecules and nonpermeable for other components of the medium. It is solid and has nanoglobular structure. Thus, gold film deposited to the surface of sensitive layer acts as a filter, which provides required degree of sensor selectivity. Besides, it is used as second electrode for conventional sandwich structures. The use of coplanar system, composed of two nickel electrodes, in the sensor construction enables to refuse from external electrical contact with gold film. This increases a reliability of the device as a whole and simplifies its production technology.

Morphology of the layer surface has been studied with Solver-P47 scanning probe microscope, and adsorption properties - by water vapor adsorption isotherms. The isotherms of adsorption of water vapors at the sensor sensitive layer have been plotted by quartz crystal microbalance. The study has been conducted with the use of AT-cut quartz crystal resonators with natural resonance frequency of 5.0, 8.0, 9.0 and 9.1 MHz. The "Rodnik" and "Rodnik-2" moisture generators have been used to prepare the air with specified moisture content.

\section{Results}

Concentration of hydroxyl groups in a film depends mainly on the moisture content and temperature of the medium, in which it forms and/or is subjected to subsequent annealing, and its storage conditions and duration after heat treatment. Maximum layer nanoporosity is determined by the composition of tetraethoxysilane-based solution and process conditions of sensitive layer formation. The layer has pronounced nanoglobular structure with average size of globules of $1.5-2 \mathrm{~nm}$ (see Fig. 2). The surface is poorly textured; therefore, it does not disturb integrity of $10 \mathrm{~nm}$ gold film deposited on it.

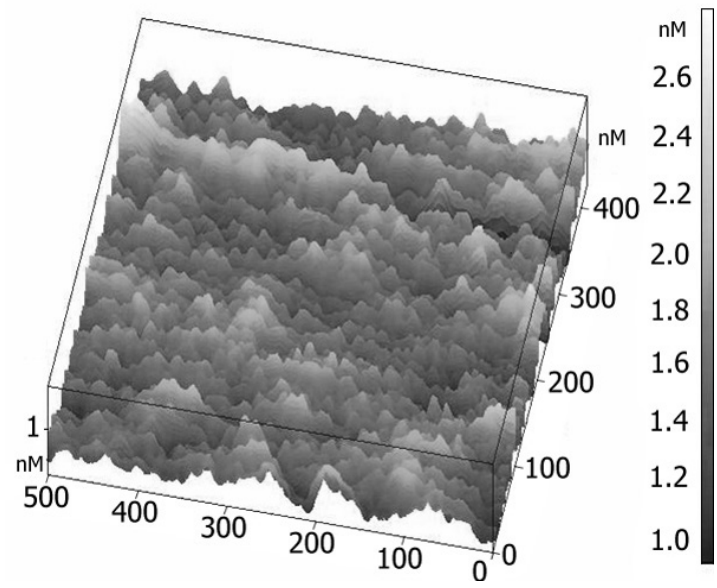

Fig. 2. SEM image of silica film's surface morphology.

Film mass increased at once as film had been deposited on the wafer. It connected with chemical sorption of moisture from environment. However, mass decreased in $60-$ $360 \mathrm{~s}$, that fact was explained by predominance 
of desorption processes of solvent and hydrolysis products out of the film. Velocity of moisture sorption and relative mass value depended on film content. Putting into film hygroscopic additives with concentration more than $5-10 \%$ mass resulted in more absolute and quick hydrolytic polycondensation of TEOS in comparison with undoped film.

The process of move off solvent and hydrolysis products is more intense during first day after deposition. This fact doesn't depend on film content. The process of heat treatment was smooth up $323 \mathrm{~K}$ to get defect-free film with good adhesion to wafer.

The heat treatment has an influence on change of hydroxyl groups, admixture content, as well as change of additives concentration in the complex composition films. We have investigated films with thickness that was equal to $40-80 \mathrm{~nm}$. Films were made from solutions based on TEOS with $5 \%$ concentration. It was determined that atom distribution in film bulk is similar for all samples. Hygroscopic additives have even distribution through film thickness.

However, segregation (increase) of concentration was moderate at surface and film/wafer interface. We determined that heat treatment at $298-573 \mathrm{~K}$ hadn't an influence on phosphorus content in the film. It was known that its distribution hadn't been depended on concentration of additive. But more complicated processes were at temperature treatment of films with $\mathrm{LiCl}$ and $\mathrm{CaCl}_{2}$. Films doped with $\mathrm{P}_{2} \mathrm{O}_{5}$ more than $5 \%$ had smallest dehydroxylation degree. Maximum layer nanoporosity is determined by the composition of TEOS-based solution and process conditions of sensitive layer formation.

So, increase of TEOS content in working solution results in growth of porosity and thickness of films at deposition. At low humidity of environment more pores formed than at high it. However, adsorption activity of doped films depends less on environment humidity, in particular for $\mathrm{P}_{2} \mathrm{O}_{5}$. Adsorption activity of the films decreases when temperature and duration of influence increase and for $\mathrm{P}_{2} \mathrm{O}_{5} / \mathrm{SiO}_{x}$ films it increase smoothly with concentration growth of additive up to $25 \%$ (see Fig. 3).

If $\mathrm{LiCl}$ is additive component then there is maximal of adsorption activity at $10 \%$ of mass content in the film. It was determined that adsorption activity of lithium chloride is more than for lithium oxide.

The value of specific surface measured for sensitive layer by linear section of adsorption isotherm plotted in the coordinates of Brunauer-
Emmett - Teller multimolecular adsorption equation is $400-450 \mathrm{~m}^{2} / \mathrm{g}$ for different samples.

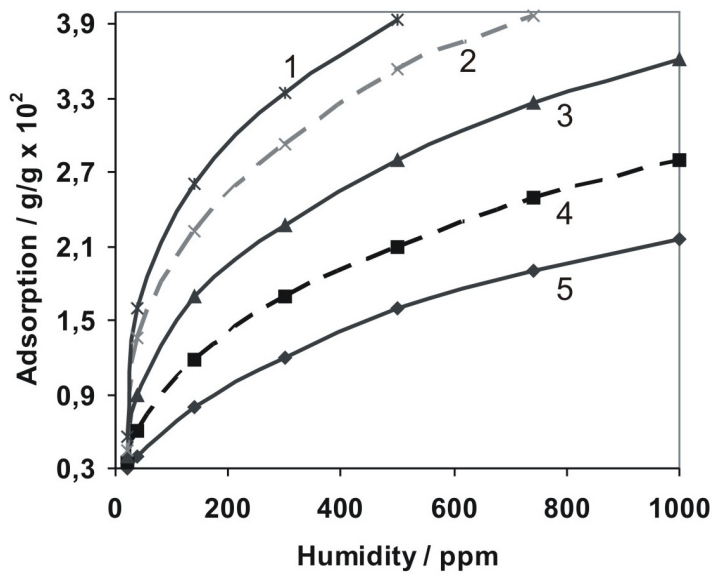

Fig. 3. The adsorption dependence of films with $10 \% \mathrm{P}_{2} \mathrm{O}_{5}$ on heating value at $293 \mathrm{~K}: 1-383 \mathrm{~K}, 1 \mathrm{~h} ; 2$ $-383 \mathrm{~K}, 4 h ; 3-453 \mathrm{~K}, 1 \mathrm{~h} ; 4-523 k, 1 h ; 5-623$ $k, 1 \mathrm{~h}$.

High sensitivity (sensitivity factor - (10-30) $\mathrm{nF} / \mathrm{K}$ by dew point) enables to use the sensor to measure humidity content for especially pure gases, used in microelectronic technology, active gases at humidity content up to $193 \mathrm{~K}$ by dew point and organic solvents (see Fig. 4). Sensor sensitivity factor is $15-35 \mathrm{nF} /{ }^{0} \mathrm{C}$ by dew point.

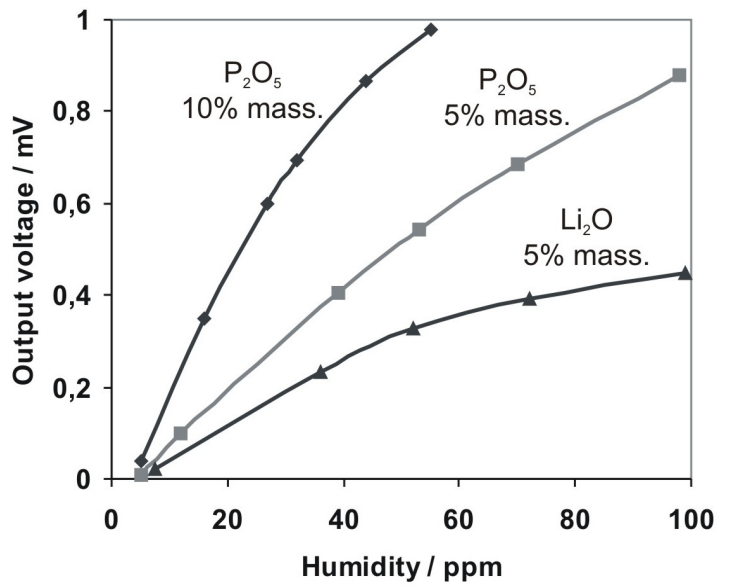

Fig. 4. The sensitivity dependence on humidity content in benzene for $\mathrm{SiO}_{x}$ films at $293 \mathrm{~K}$.

The sensor has a high dependence of sensitive layer capacity from humidity content in low humidity range. One way for taking into account temperature influence is to stabilize the high temperature of the sensor's surface at measurements in the microhumidity range. This method demands additional hardware expenditures. At the same time a range that is higher this temperature stays noncompensated. Another way is mathematical cancellation of emergent error. It doesn't influence on 
calculating power of a device and memory size. Last method was used in the present work. Special algorithm of signal processing was developed for temperature effect compensation on sensor's capacity.

Based on the results of multiple measurements, it was established that response time is 10 . $180 \mathrm{~s}$ for the microhumidity sensors (see Fig. 5 ). It depends on the level of measured trace humidity content. The sensors have hysteresis that doesn't exceed $2 \%$ in operating range.

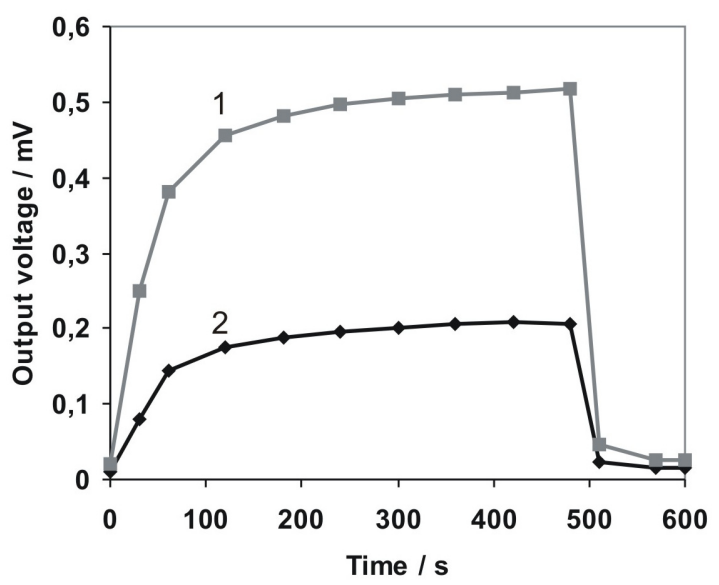

Fig. 5. Dynamic characteristics of an actuator based on film of $\mathrm{SiO}_{x}-\mathrm{P}_{2} \mathrm{O}_{5}$ (5\% mass.) in benzene at 293 K: 1 - 150 ppm; 2 - 70 ppm.

\section{Conclusion}

Concentration of hydroxyl groups in a film depends mainly on the moisture content and temperature of the medium, in which it forms and/or is subjected to subsequent annealing, and its storage conditions and duration after heat treatment. Incorporating of hygroscopic additives into $\mathrm{SiO}_{x}$ film increases the film sensitivity to water vapors. In this case, adsorption activity of $\mathrm{SiO}_{x}$ films depends on activating additive in a complex way. There are many technological factors that influence on adsorption value of films, such as: solution content, film formation conditions, temperature rate and technological environment influence.

The sensor has a high dependence of sensitive layer capacity from humidity content in low humidity range. High sensitivity enables to use the sensors to measure moisture content for especially pure gases (nitrogen, argon, oxygen, hydrogen), used in microelectronic technology, and active gases (containing singlet oxygen, chlorine, iodine, and ammonia) at moisture content up to $193 \mathrm{~K}$ by dew point.

\section{References}

[1] N. Yamazoe, Y. Shimizu, Humidity sensors: principles and application, Sensors and Actuators 10, 379-398 (1986); doi: 10.1016/02506874(86)80055-5

[2] W. Yao, X. Chen, J. Zhang, A capacitive humidity sensor based on gold-PVA core-shell nanocomposites, Sensors and Actuators B 145, 327-333 (2010); doi: 10.1016/j.snb.2009.12.021

[3] D. Saha, K.K. Mistry, R. Giri, A. Guha, K. Sensgupta, Dependence of moisture absorption property on sol-gel process of transparent nanostructured $\mathrm{y}-\mathrm{Al}_{2} \mathrm{O}_{3}$ ceramics, Sensors and Actuators B 109, 363-366 (2005); doi: 10.1016/j.snb.2005.01.002

[4] 3. S. Basu, S. Chatterjee, M. Saha, S. Bandyopadhay, K. Kr. Mistry, K. Sengupta, Study of electrical characteristics of porous alumina sensors for detection of low moisture in gases, Sensors and Actuators B 79, 182-186 (2001); doi: 10.1016/S0925-4005(01)00872-3

[5] Q. Yuan, N. Li, J. Tu, X. Li, R. Wang, T. Zhang, C. Shao, Preparation and humidity sensitive property of mesoporous $\mathrm{ZnO}-\mathrm{SiO}_{2}$ composite, Sensors and Actuators B 149, 413-419 (2010); doi: 10.1016/j.snb.2010.06.036 